\title{
Scaling-up a New Socio-mental Health Service Model in Iran to Reduce Burden of Neuropsychiatric Disorders: An Economic Evaluation Study
}

\section{Seyede Sedighe Hosseinijebeli}

Department of Health Economics, School of Health Management and Information Sciences, Iran University of Medical Sciences, Tehran. Iran

Aziz Rezapour ( $\sim$ rezapour.a@iums.ac.ir)

Health Management and Economics Research Centre, School of Health Management and Information Sciences, Iran University of Medical Sciences, Tehran.

Iran https://orcid.org/0000-0003-4032-0802

Ahmad Hajebi

Research Center for Addiction and Risky Behavior (ReCARB), Psychiatric Department, Iran University of Medical Sciences, Tehran, Iran.

Maziar Moradi Lakeh

Department of Community Medicine, Preventive Medicine and Public Health Research Centre, Iran University of Medical Sciences, Tehran, Iran

Behzad Damari

Neuroscience Institute, Tehran University of Medical Sciences, Tehran, Iran

\section{Research}

Keywords: Mental Health, Economic evaluation, Universal Health Coverage

Posted Date: August 7th, 2020

DOI: https://doi.org/10.21203/rs.3.rs-52097/v1

License: @ (i) This work is licensed under a Creative Commons Attribution 4.0 International License. Read Full License 


\section{Abstract \\ Background}

The integration of core packages of mental health care into routine primary health care has been introduced as an effective way to achieve universal health coverage in mental health care. Based on the transition of mental health care in Iran, from introducing basic mental health care in PHC to the experience of community-based mental health centers for urban areas, a new socio-mental health service model has been so far proposed. This study aimed to estimate the impact of scaling-up the new socio-mental health model at the national level as well as its associated costs.

\section{Methods}

This study was an economic evaluation following One Health Tool methodology. The data required for the study were collected in the first quarter of the year 2020 with a time horizon from 2020 to 2030 . The selected metric for summarizing health effects is healthy life years gained. Resources used in terms of drug and supply, staff salaries and outpatient visits were documented and associated costs were subsequently estimated in order to estimate the average cost of each intervention per case.

\section{Results}

The health impacts are calculated in terms of healthy life years gained for 2020-30, after adjusting the prevalence and incidence rates for each disorder. In total, 1,702,755 healthy life years were expected to be gained. Considering total 1,913,325,408 US dollars cost in base case scenario, each healthy life years gained will cost around 1124 US dollars. Based on the WHO criteria for cost-effectiveness threshold, all of the values ranged from 1042 to 1150 US dollars obtained through eight different scenarios were considered as cost-effective given the GDP per capita of 5506 US dollars for Iran in 2019.

\section{Conclusions}

The present study provided a timely analysis as the ministry of health in Iran sought to implement an ambitious plan of mental care scaling-up across the country. Therefore, this work could make a useful contribution to state-level deliberations on the implementation of this plan. Expanding such works beyond the mental health to all non-communicable diseases and making comparisons between packages of cares can be thus great evidence while the programs are aimed to be scaled-up.

\section{Introduction}

Mental health is regarded as an integral part of an individual's capacity to lead a fulfilling life. Disturbances of a person's mental well-being can thus adversely compromise this capacity as well as choices made in this respect, resulting not only in diminished functioning at individual level but also broader welfare losses for households and society [1].

Although effective interventions have been thus far introduced and affordable methods for their delivery have been shown to work, scaling-up the quality of mental health services has not occurred in most countries. Accordingly, mental health care should be included as an essential component of universal health coverage (UHC) and even fully integrated into global response to other health priorities whereas access to quality of care and financial risk protection is ensured [2].

To note, depression and anxiety are also responsible for more than $10 \%$ of the global burden of disease (GBD) and cost $\$ 1$ trillion every year in terms of lost productivity. This is accordingly a significant cost with regard to the years of (healthy) life lost (YLL) and the existing situation makes life much harder for the most vulnerable people in the world. Three quarters of the mental health disease burden is observed in low-to-middle-income countries (LMICs), and governments and households - those least able to afford it - bear the burden of mental health care costs [3].

Current coverage of essential mental health care services in LMICs is very limited. Resources made available by governments for provision of communitybased and person-centered mental health care services are often very modest. The resources that are made available are also typically directed towards more specialized and institutional services, which are not easily accessible. Without appropriate access to decent services and adequate protection, individuals with mental disorders and their families are correspondingly facing a difficult choice, namely, paying out of pocket for treatments of variable and sometimes poor quality or going without treatments altogether [4].

The economic consequences of low investments in mental health are staggering, with an estimated loss of US\$16 trillion to the global economy due to mental disorders (for the period: 2010-30), driven in part by the early age of onset and loss of productivity across the life course. In 2011, the Grand Challenges in Global Mental Health initiative led by the United States (US) National Institute of Mental Health (NIMH) prioritized implementation research questions to reduce treatment gaps for mental disorders. The first priority identified was the integration of core packages of mental health care services into routine primary health care $(\mathrm{PHC})$ ones [2].

Addressing the large and growing burden of mental, neurological, and substance use (MNS) disorders at the population level via scaled-up implementation of evidence-based treatment and prevention has been repeatedly essential over the past decade, and it can be expected to place new resource demands on the health systems of LMICs [4]. 
In Iran, about 3\% of health care expenditure is allocated to mental health. For this purpose, there are 61.2 mental health workers per 100,000 Iranians, far exceeding Iran's geographical neighbors. Outpatient facilities in this country are also active, treating nearly one in 100 of the general population while there are about 18 beds per 100,000 populations, in various settings including community-based facilities and hospitals [5].

Based on the transition of mental health care in Iran within about two decades, from introducing basic mental health care in PHC to the experience of community-based mental health centers $(\mathrm{CMHC})$ for urban areas, a new socio-mental health service model, with two basic and advanced service strata, has been so far proposed. The given model has been piloted in eight cities in this country over the last five years.

The first mental health program was piloted from 1992 to 1994 and after more than two decades, it now covers 18 million (82.8\%) rural residents and 10 million (21.7\%) citizens in urban areas. The program mainly addressed severe mental health disorders, epilepsy, and mental retardation. While it has been proven successful for villages, this program is not sufficient for those residing in urban areas. In response to the need of urban dwellers, the structure of CMHC for urban areas was designed to target those with neurotic disorders including depression and anxiety as well as severe mental disorders such as schizophrenia, bipolar disorder, and suicide attempts [6]

The new socio-mental health service model is thus comprised of two levels of health services, i.e., basic and advanced mental health services. The basic level services include public education on the basics of socio-mental health skills and screening. At this level, cases with target mental conditions are recognized by community health workers and then referred to mental health specialists for further evaluations and interventions. Using an inter-sectorial approach, patients in need of social services should be referred to a relevant service provided by other organizations in order to strengthen their social support [6].

The advanced socio-mental health services take account of facilities for mental and social emergencies and provide treatments for referrals from level one. Such services should be delivered by a collaborative care team comprised of psychologists, clinical psychiatrists, etc. at the integrated mental health settings such as PHC health centers, CMHCs, and hospitals [6].

Accordingly, scaling-up mental health services in a successful manner involves putting a range of human, physical, and other resource inputs together in order to deliver interventions and services capable of improving mental health status and related outcomes. In view of that, an essential element of evidence-based mental health service planning and scaling-up is related to an assessment of what resources are required for delivering of services to populations in need and meeting program goals [7].

Therefore, this study aimed to estimate the impact of scaling-up the new socio-mental health model at the national level as well as its associated costs to make an investment case to be considered by policy-makers for further evidence-based policy-making.

\section{Methodology}

\section{Study Design and Tools}

This study was an economic evaluation to examine the expected health gains of scaling-up certain interventions as well as their costs to reduce the burden of mental disorders in different groups of patients suffering from anxiety, depression, bipolar disorder, and epilepsy in Iran. The scaling-up scenario was thus tested against the no scaling-up scenario with the existing level of care offered in the system. The data required for the study were collected in the first quarter of the year 2020 and the time horizon was a 10-year period from 2020 to 2030 . In this study, the volume of services provided in the private sector was not estimated. It was therefore assumed that all the services were being provided by the government within the facilities of the Ministry of Health and Medical Education (MOHME) and the analysis of costs was fulfilled based on its perspective.

\section{One Health Tool Module on MNS Disorders}

The OneHealth Tool (OHT), a software tool developed by the international costing experts from the World Health Organization (WHO) and other United Nations (UN) agencies, was employed to start this study. The mental health module of the OHT was accordingly developed in order to ensure that the national mental health development plans have been carried out within a framework of assessment of overall health system capacity and to take financial sustainability and outcomes-based planning into account [8].

\section{Research Planning}

Consulting with national planners at the Department of Mental Health in the MOHME, priority MNS disorders including major depression, anxiety, bipolar disorder, and epilepsy were selected for this study. Moreover, appropriate mental health care packages and scenarios, current and target coverage levels for specific intervention strategies, and scaling-up period were identified through consultations with the expert group of national planners and program managers.

For clinical-level consideration of resource use profiles for different disorders and interventions, unit costs and prices for health care services and commodities (such as those for staff salaries, outpatient visits, and psychotropic medications), one of the pilot sites (namely, the city of Osku in East Azerbaijan Province) was selected. This pilot site was identified as the best practice to the program, benefiting from excellent documentation and assessments. To collect these data inputs, the research team, working with local team members and other national staff, identified and utilized local data sources and visited the site in a three-day tour. The data checklist adapted from the study by Chisholm [8] was also modified and used in order to facilitate and document the process of data contextualization.

\section{Data Contextualization}

\section{Health Impacts}


The selected metric for summarizing health effects at the population level is (healthy) life years (LYs) gained (equivalent to disability-adjusted life years ( DALYs) averted), where one DALY could be thought of as one YLL. (Healthy) LYs is also computed with reference to country-specific life tables that have been already built into the model, and reflect the combined time spent by the population in a particular state of health with a known degree (or free) of disability. Disability levels were drawn from the GBD 2010 study [9]. Implementing or scaling-up an effective intervention in the population was thus modeled to reduce the time spent in a disabling state, either by reducing prevalence (e.g., by decreasing the number of new cases or increasing the remission rate), or by improving the level of functioning in people with the condition in question [8].

\section{Epidemiology}

Iran has a history in mental health surveys and it is therefore possible to make use of high-quality epidemiological data from the national surveys [10, 11, 12]. Although, national estimates were available for major depression and anxiety but the GBD estimates were employed to meet age-stratified data sheet of the $\mathrm{OHT}$ on the prevalence rates for all disorders. As there were no comprehensive sources of national data available for incidence rates, the GBD estimates were used for all estimations.

\section{Estimating Resources, Costs, and Coverage Levels}

The key categories of health service costs in the $\mathrm{OHT}$ included drug and supply costs (e.g., daily dose of a generically produced first-line anti-psychotic or antiepileptic medication), costs of response to ambulatory contacts by mental health or general health workers (such as psychologists, counselors, and community health workers), and costs of hospital-based outpatient/inpatient care services. In addition, program-level resource needs were identified, including overall program management and administration as well as training [8].

\section{Intervention Costing}

Resources used in terms of drug and supply, staff salaries, and outpatient visits were documented through field visits to the pilot site and the associated costs were subsequently estimated based on accounting documents available in the program management office in order to estimate the average cost of each intervention per case.

As described in the introduction of the new care model, basic package of care (e.g. basic psychosocial treatment plus medication therapy of moderate cases of anxiety/depression) were being provided in health care facilities and severe cases of those disorders in addition to all bipolar cases could be referred to the CMHCs for intensive care and social support. It is noteworthy that the WHO Mental Health Gap Action Program (mhGAP) guideline was practiced with slight changes in real time spent and availability of recommended drugs in the whole system of integrated mental health care in PHC.

All three levels of care (health care facility, $\mathrm{CMHC}$, and general hospital (psychiatric ward)) were accordingly visited and all staff including community health workers, socio-mental health experts, general practitioners, psychologists, psychiatrists, and social workers were interviewed in order to identify activities and estimate time spent for each patient and intervention. The drug and supply list used for each intervention was also documented and the price list was acquired from the health information system (HIS) of the hospital with a particular focus on most-prescribed medications available in the primary care setting. It should be noted that drug and supply prices have the same tariffs across the country in public hospitals and the PHC network.

\section{Program Costing}

Information on the program-specific staff required for scaling-up were obtained from background strategic documents of the program available at the Department of Mental Health.

\section{Total Cost}

The total costs of scaling-up an intervention in a given year for a country was thus derived by multiplying resource use needs by their respective unit costs to give an average cost per case. It was then multiplied by the total number of cases, expected to receive a particular intervention (given by the prevalence of the disorder multiplied by the rate of treatment coverage of specific intervention strategies in the population), that is, total cost $=$ population $\times$ prevalence rate $\times$ coverage $\times$ treatment cost per case [8]. To estimate the costs, the expected inflation rate for the period: 2020-2030 was applied using extrapolation based on the actual inflation rates for 2010-19. Since all the costs were calculated in Iranian Rial (IRR), to convert the costs in US\$, the exchange rate for the study period was estimated through extrapolation based on the UN exchange rates for the past 10 years.

\section{Coverage}

The estimates of the baseline coverage were obtained from expert opinions based on the coverage of health care facilities per population. Given the expected challenges of scaling-up the new model at the national level, a modest coverage target of $30 \%$ by 2030 was set for the intensive packages of care and $70 \%$ target coverage was considered for the basic package, which have already had $40 \%$ baseline coverage.

\section{Assumptions}

In this study, the volume of services provided in the private sector was not estimated. It was assumed that all the services had been provided by the government within the facilities of MOHME. Besides the base case scenario which is provided below, we examined seven others scenarios in order to provide a sensivity analysis.

\section{Results}


Table 1

Intervention coverages - Front Loaded pattern

\begin{tabular}{|c|c|c|c|c|c|c|c|c|c|c|c|}
\hline Scale up period & 2020 & 2021 & 2022 & 2023 & 2024 & 2025 & 2026 & 2027 & 2028 & 2029 & 2030 \\
\hline \multicolumn{12}{|l|}{ Anxiety disorders } \\
\hline $\begin{array}{l}\text { Basic psychosocial treatment and anti-depressant } \\
\text { medication for anxiety disorders }\end{array}$ & 40 & 43.9 & 47.5 & 50.9 & 54.1 & 57.1 & 59.9 & 62.6 & 65.2 & 67.7 & 70 \\
\hline $\begin{array}{l}\text { Intensive psychosocial treatment and medication therapy for } \\
\text { anxiety disorders }\end{array}$ & 1 & 12.6 & 17.3 & 20.4 & 22.6 & 24.4 & 25.8 & 27.1 & 28.2 & 29.1 & 30 \\
\hline \multicolumn{12}{|l|}{ Depression } \\
\hline $\begin{array}{l}\text { Basic psychosocial treatment and anti-depressant } \\
\text { medication of first episode cases }\end{array}$ & 40 & 43.0 & 46.0 & 49.0 & 52.0 & 55.0 & 58.0 & 61.0 & 64.0 & 67.0 & 70 \\
\hline $\begin{array}{l}\text { Intensive psychosocial treatment and anti-depressant } \\
\text { medication of first episode cases }\end{array}$ & 1 & 3.9 & 6.8 & 9.7 & 12.6 & 15.5 & 18.4 & 21.3 & 24.2 & 27.1 & 30 \\
\hline $\begin{array}{l}\text { Intensive psychosocial treatment and anti-depressant } \\
\text { medication of moderate-severe cases on a maintenance } \\
\text { basis }\end{array}$ & 1 & 3.9 & 6.8 & 9.7 & 12.6 & 15.5 & 18.4 & 21.3 & 24.2 & 27.1 & 30 \\
\hline \multicolumn{12}{|l|}{ Bipolar disorder } \\
\hline $\begin{array}{l}\text { Intensive psychosocial intervention for bipolar disorder, plus } \\
\text { mood-stabilizing medication }\end{array}$ & 1 & 12.6 & 17.3 & 20.4 & 22.6 & 24.4 & 25.8 & 27.1 & 28.2 & 29.1 & 30 \\
\hline \multicolumn{12}{|l|}{ Epilepsy } \\
\hline $\begin{array}{l}\text { Basic psychosocial support, advice, and follow-up, plus anti- } \\
\text { epileptic medication }\end{array}$ & 40 & 43.9 & 47.5 & 50.9 & 54.1 & 57.1 & 59.9 & 62.6 & 65.2 & 67.7 & 70 \\
\hline
\end{tabular}

After consulting the national managers of the program, the baseline coverage levels (2020) as well as the target ones (2030), were defined for each of the interventions. Among different scaling-up patterns available in the tool including linear, S-shaped, front-loaded, and exponential, the front-loaded interpolation pattern, recommended for middle-income countries was chosen. The coverage levels for all the interventions in each year are presented in Table 1.

\begin{tabular}{|c|c|c|c|c|c|c|c|c|}
\hline & & & & & & $\begin{array}{c}\text { Table } 2 \\
\text { Total cost }\end{array}$ & & \\
\hline & 2020 & 2021 & 2022 & 2023 & 2024 & 2025 & 2026 & 2027 \\
\hline $\begin{array}{l}\text { Intervention } \\
\text { costs }\end{array}$ & $35,671,581.38$ & $93,650,099.74$ & $123,439,284.68$ & $146,376,491.90$ & $166,162,736.49$ & $184,106,179.49$ & $200,830,413.70$ & $216,638,37^{\circ}$ \\
\hline $\begin{array}{l}\text { Programme } \\
\text { costs }\end{array}$ & $141,574.60$ & $301,207.19$ & $424,001.49$ & $560,519.86$ & $683,314.16$ & $819,832.53$ & $942,626.83$ & $1,051,697.0$ \\
\hline Total costs & $35,813,155.99$ & $93,951,306.93$ & $123,863,286.17$ & $146,937,011.76$ & $166,846,050.65$ & $184,926,012.02$ & $201,773,040.52$ & $217,690,07$ ، \\
\hline Per Capita & 23.05 US\$ & & & & & & & \\
\hline
\end{tabular}

The total cost including both intervention and program costs for all interventions selected from the module of mental and neurological disorders are illustrated in Table 2. To note, all the cost values were converted into the US dollars for ease of interpretation and comparison, but local currency values were used in the context of ongoing policy dialogue. The exchange rate was captured from the UN exchange rates in June 2020.

Table 3

Healthy Years Gained

\begin{tabular}{|llllllllllll|l|}
\hline Impact scale up & $\mathbf{2 0 2 0}$ & $\mathbf{2 0 2 1}$ & $\mathbf{2 0 2 2}$ & $\mathbf{2 0 2 3}$ & $\mathbf{2 0 2 4}$ & $\mathbf{2 0 2 5}$ & $\mathbf{2 0 2 6}$ & $\mathbf{2 0 2 7}$ & $\mathbf{2 0 2 8}$ & $\mathbf{2 0 2 9}$ & $\mathbf{2 0 3 0}$ & Total \\
\hline Depression & 0 & 35,873 & 59,717 & 78,750 & 95,490 & 110,979 & 125,688 & 139,854 & 153,611 & 167,043 & 180,231 & 1147236 \\
\hline Anxiety & 0 & 8,235 & 13,966 & 19,595 & 25,477 & 31,699 & 38,272 & 45,177 & 52,380 & 59,847 & 67,544 & 362192 \\
\hline Bipolar & 0 & 4,461 & 6,371 & 7,658 & 8,660 & 9,498 & 10,229 & 10,884 & 11,480 & 12,027 & 12,534 & 93802 \\
\hline Epilepsy & 0 & 1,691 & 3,423 & 5,199 & 7,018 & 8,879 & 10,774 & 12,698 & 14,645 & 16,610 & 18,588 & 99525 \\
\hline Total & $\mathbf{1 , 7 0 2 , 7 5 5}$ & & & & & & & & & \\
\hline
\end{tabular}

As shown in Table 3, the health impacts are calculated in terms of (healthy) LYs gained for 2020-30, after adjusting the prevalence and incidence rates for each disorder in the NCD impact module. Depression has the biggest share in the health impact, as it is the most prevalent mental disorder in Iran. In total, $1,702,755$ (healthy) LYs were expected to be gained via scaling-up the program at national levels. Considering the total $1,913,325,408$ US dollars cost, each (healthy) LY gained will cost around 1124 US dollars. 
Table 4

Sensivity Analysis

\begin{tabular}{|c|c|c|c|c|c|c|c|}
\hline \multirow[t]{2}{*}{ Scenarios } & \multirow{2}{*}{$\begin{array}{l}\text { Base case(Front } \\
\text { loaded) }\end{array}$} & \multicolumn{2}{|l|}{ Scale Up Pattern } & \multicolumn{2}{|c|}{ Epidemiologic data Cls } & \multicolumn{2}{|c|}{ Expected Coverage Levels } \\
\hline & & Exponential & Linear & Lower & Upper & Coverage 1 & Coverage 2 \\
\hline $\begin{array}{l}\text { Intervention } \\
\text { costs }\end{array}$ & $1,904,589,150.63$ & $1,284,206,137.42$ & $1,606,994,204.96$ & $1,506,982,112.94$ & $2,407,299,182.00$ & $2,047,051,802.12$ & $2,479,268,153.54$ \\
\hline $\begin{array}{l}\text { Program } \\
\text { costs }\end{array}$ & $8,736,258.21$ & $8,736,258.21$ & $8,736,258.21$ & $8,736,258.21$ & $8,736,258.21$ & $8,736,258.21$ & $8,736,258.21$ \\
\hline Total costs & $1,913,325,408.84$ & $1,292,942,395.63$ & $1,615,730,463.17$ & $1,515,718,371.15$ & $2,416,035,440.21$ & $2,055,788,060.33$ & $2,488,004,411.75$ \\
\hline \multicolumn{8}{|c|}{ Impact scale up } \\
\hline Depression & $1,147,236$ & 753131 & 957688 & 934,294 & $1,403,864$ & $1,259,254$ & $1,638,144$ \\
\hline Anxiety & 362,192 & 282307 & 324450 & 316,006 & 418,867 & 368,865 & 520,404 \\
\hline Bipolar & 93,802 & 38658 & 67133 & 65,494 & 130,647 & 93,802 & 128,165 \\
\hline Epilepsy & 99,525 & 88300 & 94216 & 61,126 & 150,542 & 66,251 & 101,994 \\
\hline $\begin{array}{l}\text { Total } \\
\text { Impact }\end{array}$ & 1702755 & 1162396 & 1443487 & 1376920 & 2103920 & 1788172 & 2388707 \\
\hline $\begin{array}{l}\text { Cost per a } \\
\text { Healthy LY }\end{array}$ & 1124 & 1112 & 1119 & 1101 & 1148 & 1150 & 1042 \\
\hline
\end{tabular}

In order to provide a sensivity analysis, we have examined three sets of scenarios changing the scale up patterns, investigating the lower and upper bound of Confidence Intervals (Cls) for prevalence and incidence rates and finally changing the coverage levels.

As shown in the Table 4, the cost per a healthy LY changes from 1112 US\$ in exponential pattern to 1119 in linear one. Based on the health system development level in Iran, the front loaded pattern, recommended for middle income countries, is selected and other scenarios are tested against it.

Then, in the next set of scenarios, the epidemiological data, obtained from GBD estimates, were changed using the Cls of reported prevalence and incidence rates resulting in a range of 1101 to 1148 US dollars per each healthy LY gained.

Finally, we have examined different coverage levels as the main driver for costs and impacts. In the scenario "Coverage 1", we have changed the existing coverage of basic interventions from $40-50 \%$ while in the "Coverage 2" scenario the target level for intensive care were adjusted for $40 \%$ instead of $30 \%$ in base case scenario. Moreover, in the scenario "Coverage 3 " the target level for the basic package were reduced considering $60 \%$ level of coverage.

Based on the WHO criteria [13], all of these values ranged from 1042 to 1150 US\$ obtained through eight different scenarios were considered as cost-effective.

\section{Discussion}

In the face of large and increasing burden of mental diseases and treatment gaps existing in Iran, a new socio-mental health service model was introduced to the mental health system of this country. This program, available in PHC settings, could significantly contribute to achieving the goals of the UHC for the MNS disorders in terms of financial protection and service provision. This study was further concerned with informing national policy-makers about resource needs and costs of scaling-up such mental health services in national level.

Mental health budget in Iran equals to $3 \%$ of total health expenditure [5], which is relatively low considering the share of the MNS disorders in the national burden of diseases. Convincing high-level policy-makers to increase the share of mental health budget accordingly needs studies reflecting on the expected health gains and the associated costs, as a valuable input for evidence-based policy-making.

The OHT methodology used by Chisholm [8] was followed in this study. In comparison with their estimation for five LMICs, the results of this study indicated that the resource needs for scaling-up mental health services did not need to be substantial. The per capita cost of scaling-up the new socio-mental health service model in Iran was accordingly estimated about 23.05 US dollars.

Although differences in health system development, care packages, and costing requirements make it much difficult to compare results between various countries in such studies, the cost estimated here for Iran was 1124 US dollars in terms of cost per (healthy) LY gained. This value was far less than what was estimated for South Africa, around 18000 US dollars, whose per capita income was comparable to that of Iran as a middle-income country. This estimation was somehow analogous to that of India with 761 US dollars per (healthy) LY gained from scaling-up their mental health program [8].

Through examining different scenarios, it was shown that achieving more coverage level in new socio-mental health in terms of increased level of intensive care package (increasing the expected level from 30-40\%) could significantly reduce the cost per each (healthy) LY gained from 1124 US\$ in base case compared to 1042 US\$ in respective scenario.

Furthermore, based on the WHO criteria for cost-effectiveness threshold [13], cost per (healthy) LY gained (i.e., DALY averted) was considered very costeffective if it was less than a gross domestic product (GDP) per capita in a specified country. The estimation of 1124 US dollars per (healthy) LY gained 
through the scaling-up period of the new socio mental health care could be regarded very cost-effective given the GDP per capita of 5506 US dollars for Iran in 2019.

Therefore, the health returns on such an investment are substantial. Such information on the costs and health impacts of scaling-up could further provide important evidence that could be used in dialogues with health planners and policy-makers at the national level, particularly in the context of increased policy attention to the rising burden of mental and neurological disorders.

The estimates reported herein additionally represented an initial set of projections, based on national available evidence and informed inputs of local experts; however, such estimates are subjected to further discussions, reviews, and revisions as planning cycles and political processes are evolving. New projections will be accordingly prepared in the light of changes to policies and plans, such as revised target coverage levels or lengthened implementation periods.

\section{Conclusion}

Using the $\mathrm{OHT}$ strategic planning could help policy-makers with evidence-informed policy- making to prioritize cost-effective interventions and to improve allocative efficiency. The present study provided a timely analysis as the Department of Mental Health in Iran sought to implement an ambitious plan of mental health scaling-up across the country, collaborating with the MOHME and the Ministry of Interior. Therefore, this work could make a useful contribution to state-level deliberations on the implementation of this plan at national level. Expanding such works beyond the mental health to all non-communicable diseases (NCDs) and making comparisons within and between specific packages of cares can be thus great evidence while the programs are developing at different departments aimed to be scaled-up at national and sub-national levels.

\section{Limitations}

Working with the OHT through different modules can be a challenging job with hundreds of parameters to be revised and updated. A broad concern is also related to the available national evidence on a number of domains. These include epidemiological data on the burden of the MNS disorders, extent of current coverage and expenditure, as well as evidence base for locally adapted cost-effective interventions. While regional and global default estimates are available, the tool developers strongly encourage updating parameters with national estimates.

To update the prevalence and incidence rates, the GBD estimates were accordingly used as the last national mental health survey of Iran did not include estimates on the incidence rates while the prevalence rates had been also limited to major depression and anxiety. In case of cost- effectiveness of interventions and transition rates (e.g. remission and mortality rates), the default $\mathrm{OHT}$ rates were employed, since there were no national estimates or even studies investigating such parameters.

\section{Declarations}

\section{Ethics approval and consent to participate}

Not Applicable

\section{Consent for publication}

Not Applicable

\section{Availability of data and materials}

The datasets used and/or analyzed during the current study are available from the corresponding author on reasonable request.

\section{Competing interests}

The authors declare that they have no competing interests.

\section{Funding}

This study is supported by and approved in the School of health management and information science in Iran university of medical sciences as a PhD thesis by Ms Seyede Sedighe Hosseini Jebeli. Registration number: IR.IUMS.REC.1397.050

\section{Authors' contributions}

SHJ have proposed the topic and the study was designed by SHJ and AR. The study protocol was finalized in collaboration with MML and AH. The epidemiological data was gathered by SHJ, MML and AH while SHJ, AR and BD accomplished the costing part. The data were analyzed by SHJ and then critically interpreted by $\mathrm{AR}, \mathrm{MML}$ and $\mathrm{AH}$. SHJ drafted the paper and all authors reviewed it accordingly and have provided their input.

\section{Acknowledgements}


We would like to express our sincere gratitude to Mr Vahab Asl Rahimi, Manager of the socio- mental health department and Mrs Azam Hemati, mental health expert and all colleagues at the Tabriz university of medical sciences who supported us both technically and administratively in order to collect data to accomplish this project.

\section{References}

1. -World Health Organization. Investing in mental health: evidence for action.

2. -Patel V, Saxena S, Lund C, Thornicroft G, Baingana F, Bolton P, Chisholm D, Collins PY, Cooper JL, Eaton J, Herrman H. The Lancet Commission on global mental health and sustainable development. The Lancet. 2018 Oct;27(10157):1553-98. 392(.

3. -Caddick H, Horne B, Mackenzie J, Tilley H. Investing in mental health in low-income countries. London: Overseas Development Institute; 2016 Dec.

4. -Chisholm D, Docrat S, Abdulmalik J, Alem A, Gureje O, Gurung D, Hanlon C, Jordans MJ, Kangere S, Kigozi F, Mugisha J. Mental health financing challenges, opportunities and strategies in low-and middle-income countries: findings from the Emerald project. BJPsych open. 2019 Sep;5(5).

5. -World Health Organization. WHO-AIMS report on mental health system in the Islamic Republic of Iran. Tehrran: World Health Organization-Assessment Instrument for Mental Health Systems; 2006.

6. -Damari B, Alikhani S, Riazi-Isfahani S, Hajebi A. Transition of mental health to a more responsible service in Iran. Iranian journal of psychiatry. 2017 Jan;12(1):36.

7. -Chisholm D, Burman-Roy S, Fekadu A, Kathree T, Kizza D, Luitel NP, Petersen I, Shidhaye R, De Silva M, Lund C. Estimating the cost of implementing district mental healthcare plans in five low-and middle-income countries: the PRIME study. The British Journal of Psychiatry. 2016 Jan;208(s56):s71-8.

8. -Chisholm D, Heslin M, Docrat S, Nanda S, Shidhaye R, Upadhaya N, Jordans M, Abdulmalik J, Olayiwola S, Gureje O, Kizza D. Scaling-up services for psychosis, depression and epilepsy in sub-Saharan Africa and South Asia: development and application of a mental health systems planning tool (OneHealth). Epidemiology and Psychiatric Sciences. 2017 Jun;26(3):234-44.

9. -Salomon JA, Vos T, Hogan DR, Gagnon M, Naghavi M, Mokdad A, Begum N, Shah R, Karyana M, Kosen S, Farje MR. Common values in assessing health outcomes from disease and injury: disability weights measurement study for the Global Burden of Disease Study 2010. The Lancet. 2012

Dec;15(9859):2129-43. 380(.

10. -Noorbala AA, Faghihzadeh S, Kamali K, Yazdi SA, Hajebi A, Mousavi MT, Akhondzadeh S, Faghihzadeh E, Nouri B. Mental health survey of the Iranian adult population in 2015. Archives of Iranian medicine. 2017 Mar 1;20(3):0-.

11. -Sharifi V, Amin-Esmaeili M, Hajebi A, Motevalian A, Radgoodarzi R, Hefazi M, Rahimi-Movaghar A. Twelve-month prevalence and correlates of psychiatric disorders in Iran: the Iranian Mental Health Survey, 2011. Archives of Iranian medicine. 2015 Feb 1;18(2):0-

12. -Rahimi-Movaghar A, Sharifi V, Motevalian A, Amin-Esmaeili M, Hadjebi A, Rad Goodarzi R. Iranian National Mental Health Survey: prevalence, severity and costs of mental disorders and service utilization (IranMHS). Tehran: Mehrsa; 2014.

13. -Bertram MY, Lauer JA, De Joncheere K, Edejer T, Hutubessy R, Kieny MP, Hill SR. Cost-effectiveness thresholds: pros and cons. Bulletin of the World Health Organization. 2016 Dec 1;94(12):925. 